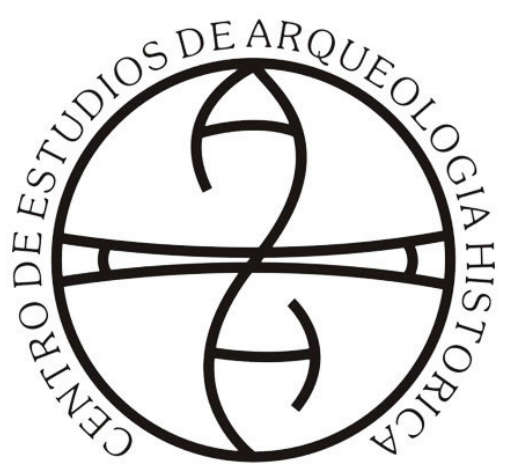

Centro de Estudios de Arqueología Histórica

Universidad Nacional de Rosario

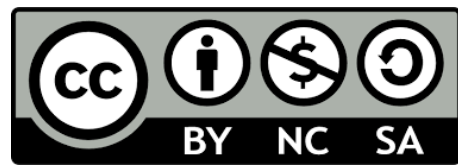

Teoría y Práctica de la Arqueología Histórica

Latinoamericana | Año IX, Volumen 10 | 2020

Revista del Centro de Estudios de Arqueología Histórica, Facultad de Humanidades y Artes,

Universidad Nacional de Rosario

https://teoriaypracticaah.unr.edu.ar/index.php/index

https://rephip.unr.edu.ar/handle/2133/14804

ISSN en línea: 2591-2801

ISSN versión impresa: 2250-866X

Atribución-NoComercial-CompartirIgual 4.0 Internacional (CC BY-NC-SA 4.0)

https://creativecommons.org/licenses/by-nc-sa/4.0/deed.es

Pablo José Pifano (ID.: https://orcid.org/0000-0002-

0193-656X) y María Cecilia Páez (ID.: https://orcid. org/0000-0001-6405-9202). Aproximación cronológica al funcionamiento del molino hidráulico de Payogasta (Cachi, Salta) durante los siglos XIX y XX

\title{
Aproximación CRONOLÓgICA AL FUNCIONAMIENTO DEL molino hidráulico de Payogasta (Cachi, Salta) DURANTE LOS SIGLOS XIX Y XX
}

\section{Chronological approach to the operation of Payogasta's hydraulic mill (CACHI, Salta) during The XIX ANd XX CEnTURIES}

\author{
Pablo José Pifano* y María Cecilia Páez**
}

\section{Resumen}

El antiguo molino harinero localizado en el Municipio de Payogasta (Salta, Argentina) se ubica en la margen derecha del río Calchaquí. Se trata de un conjunto de estructuras de adobe (seis habitaciones relevadas hasta el momento), la mayoría de las cuales aún conservan los techos. La maquinaria de molienda se encuentra en una de ellas, que destaca por su buena preservación.

En este trabajo nos proponemos abordar la cronología de funcionamiento del molino, desde un enfoque transdisciplinar que ponga en diálogo diferentes registros de información: relatos orales, evidencia arqueológica y documentos históricos. Las primeras conclusiones sugieren que la estructura de molienda habría funcionado de manera ininterrumpida desde mediados del siglo XIX hasta avanzado el siglo XX. Palabras clave: transdisciplinariedad, arqueología, antropología e historia , molino harinero, Payogasta.

* Consejo Nacional de Investigaciones Científicas y Técnicas. División Arqueología, Museo de Ciencias Naturales, Facultad de Ciencias Naturales y Museo. Universidad Nacional de La Plata, Paseo del Bosque s/n, B1900FWA, La Plata, Argentina. Email: pablopifano12.91@gmail.com

** Consejo Nacional de Investigaciones Científicas y Técnicas. División Arqueología, Museo de Ciencias Naturales, Facultad de Ciencias Naturales y Museo. Universidad Nacional de La Plata, Paseo del Bosque s/n, B1900FWA, La Plata, Argentina. Email: ceciliapaez@gmail.com 
Teoría y Práctica de la Arqueología Histórica Latinoamericana

Año IX, Volumen 10 | 2020 / ISSN en línea: 2591-2801 | ISSN versión impresa: 2250-866X

\begin{abstract}
The ancient hydraulic flour mill of Payogasta (Salta, Argentina), settled on the right bank of the Calchaquí river is a set of adobe structures (six rooms have been examined so far), most of which still retain the ceilings. The grinding machinery is located in one of the best-preserved rooms.

In this paper, we intend to address the chronology of the mill, particularly the chronology of its operation, from a transdisciplinary approach that puts into dialogue different records of information: oral accounts, archaeological evidence and historical documents. The first conclusion we can make is that the grinding structure has been functioning uninterruptedly since the mid-19th century to the late 20th century.

Key words: transdisciplinary, archeology, anthropology and history, flour mill- Payogasta.
\end{abstract}

\title{
Introducción
}

La producción supradoméstica de harinas en el sector norte del valle Calchaquí (Salta, Argentina) fue una actividad preponderante para la estructura económica local y regional durante los siglos XIX y $\mathrm{XX}$. Las fuentes documentales y las entrevistas con los pobladores locales ponen de relieve el lugar que habrían tenido los molinos hidráulicos en la región, destacándose diferentes instalaciones en las localidades de Palermo, Payogasta, Cachi, Cachi Adentro,Escalchi, Laxi, Seclantas, Molinos, Colomé, Luracatao, Angastaco y Piul (Marinangeli,Plastine Pujadas y Páez,2016; Pifano y Dabadié, 2016).

La estructura de encomienda instalada una vez finalizadas las Guerras Calchaquíes dio lugar a las haciendas en el siglo XVIII, donde se destinaron grandes extensiones a la producción agrícola, con cultivos como maíz, trigo y alfalfa, además de la actividad ganadera (Hocsman, 2003; Lera, 2005; Marinangeli y Páez, 2019; Mata de López, 2005; Pais, 2011). El procesamiento de los granos para el consumo doméstico y la comercialización regional y con países vecinos como Perú, Bolivia y Chile, hizo necesaria la instalación de molinos, que funcionaban a partir de la fuerza hidráulica.

Durante el siglo XIX, la molienda de granos adquirió un carácter comercial sin precedentes, tanto dentro como fuera del valle, siendo la harina de trigo la más importante, aunque también se producía de maíz y papa helada. El trigo se adaptó rápidamente a la región, por lo que la mayor parte de las fincas contaban con su propio molino harinero. No obstante, la competencia con el puerto de Buenos Aires durante el siglo XX, junto con la expansión del ferrocarril habrían ocasionado el descenso de la producción de trigo, ya que la harina pampeana se consideraba de mayor calidad, impactando así en la actividad harinera (Lera, 2005). Para la década de 1980 aún funcionaban algunos molinos en la zona, destinados fundamentalmente a la producción doméstica o al comercio con parajes rurales (Cieza, 2010).

La información documental relevada hasta el momento sugiere la importancia de los molinos en la región desde el siglo XVIII, aunque la mayor cantidad de instalaciones corresponden al siglo siguiente. Para la provincia de Salta, hay registros de los años 1754, 1777, 1824, 1832, 1834, 1846 y 1850 y en 1855 se registra el primer molino hidráulico de la localidad de Payogasta, en el departamento de Cachi(Schlech, 1914; Pifano y Ermili,2020).

En Payogasta,actualmente se identifican al menos tres molinos, localizados en la finca de Laxi, de Ruiz de Los Llanos y el que se ubica en el pueblo viejo, sobre la vera del río Calchaquí. En este trabajo nos concentraremos en este último, formado por la maquinaria de molienda y un conjunto de habitaciones asociadas,con el objetivo de situar temporalmente la actividad de molienda que se realizaba allí. Partiendo del carácter fragmentario de la información disponible, se torna importante abordar nuestro interés de investigación a partir de un enfoque transdisciplinario que tenga en cuenta, en conjunto, el registro

Aproximación cronológica al funcionamiento del molino hidráulico de Payogasta

P. J. Pifano y M. C. PÁEZ

(Cachi, Salta) durante los siglos XIX y XX

Páginas 45-57 
arqueológico, etnohistórico y etnográfico. Tal como plantea Castro Gómez (2007) este tipo de aproximación permite relacionar los elementos y “(...) conocimientos que la modernidad había declarado como dóxicos (...)"(p.87). A diferencia de la multidisciplinariedad (que favorece la sumatoria de disciplinas) y la interdisciplinariedad (que promueve el pasaje epistemológico y metodológico de una disciplina a otra), en este caso se apela a una lógica inclusiva, que trabaje sobre los nexos y relaciones que las constituyen (Martínez Miguélez, 2007). Esto implica que un problema de investigación pueda ser abordado desde diferentes disciplinas (Molina y Vedia, 2016), lo que genera, no una adición de información, sino un conocimiento generado a partir de la relación dialógica entre ellas.

\section{El molino hidráulico del pueblo histórico de Payogasta}

El primer acercamiento al molino fue en el año 2016, en el marco de la tesis doctoral del primer autor $^{(1)}$. La estructura de molienda se ubica a los $25^{\circ} 03^{\prime} 01.4^{\prime} \mathrm{S} ; 6^{\circ} 06^{\prime} 17.5^{\prime \prime} \mathrm{W}$, en el sector conocido como "la parte antigua del pueblo de Payogasta", que fue el centro de las actividades y la circulación de bienes antes del traslado de los principales edificios públicos a la localización actual(Figura 1). Esta modificación respondió al interés de conformar el pueblo en torno al paso de la ruta 40, que se comenzó a construir en 1935.

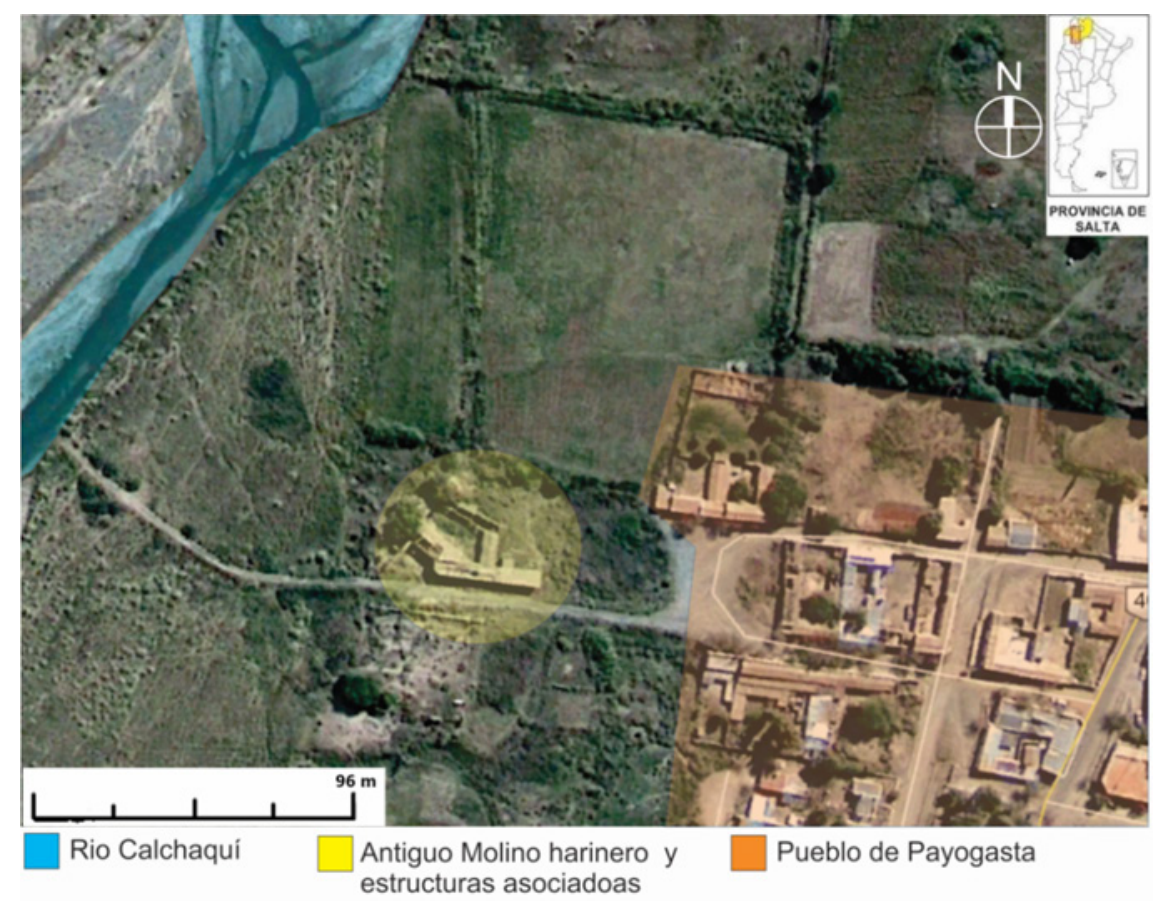

Figura 1. Ubicación del antiguo molino en relación al río Calchaquí y a la localización actual del pueblo de Payogasta. 
El molino harinero de Payogasta cuenta con la habitación de molienda y otras cinco estructuras asociadas, las que habrían tenido diferentes funciones vinculadas con la molienda como, por ejemplo, almacenar bolsas de harina, hospedar a los visitantes, brindar espacios de reunión, herrería, entre otras (Pifano y Ermili, 2020). Todas las habitaciones relevadas tienen un cimiento de cantos rodados de tamaño variable con barro y un sobrecimiento de ladrillos de adobe (Figura 2).

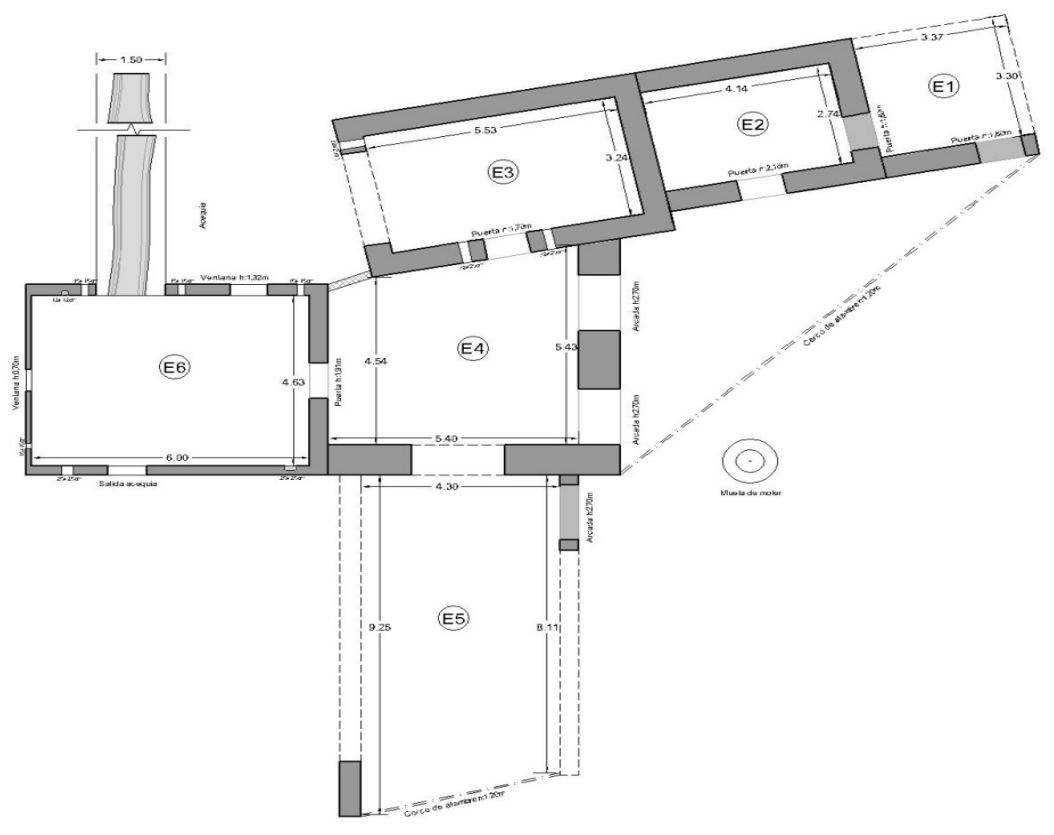

Figura 2. Plano del edificio. Se puede observar la habitación de molienda (E6) y habitaciones asociadas (E1, E2, E3, E4, E5).

La habitación de molienda (E6) se encuentra en buen estado de conservación, tanto a nivel interno como externo. Al interior de la misma se halla la maquinaria de molienda: ambas muelas de moler, la solera (inferior y fija) y la volantera (móvil y por encima de la anterior). Por arriba de las muelas está la tolva de madera, con su típica forma piramidal invertida, donde se depositan los productos de la actividad, además de herramientas específicas para la realización del trabajo. Tal es el caso de una pala manual identificada en el lugar para mover la harina depositada, y el almud de madera que es la unidad de medida (Figura 3). 


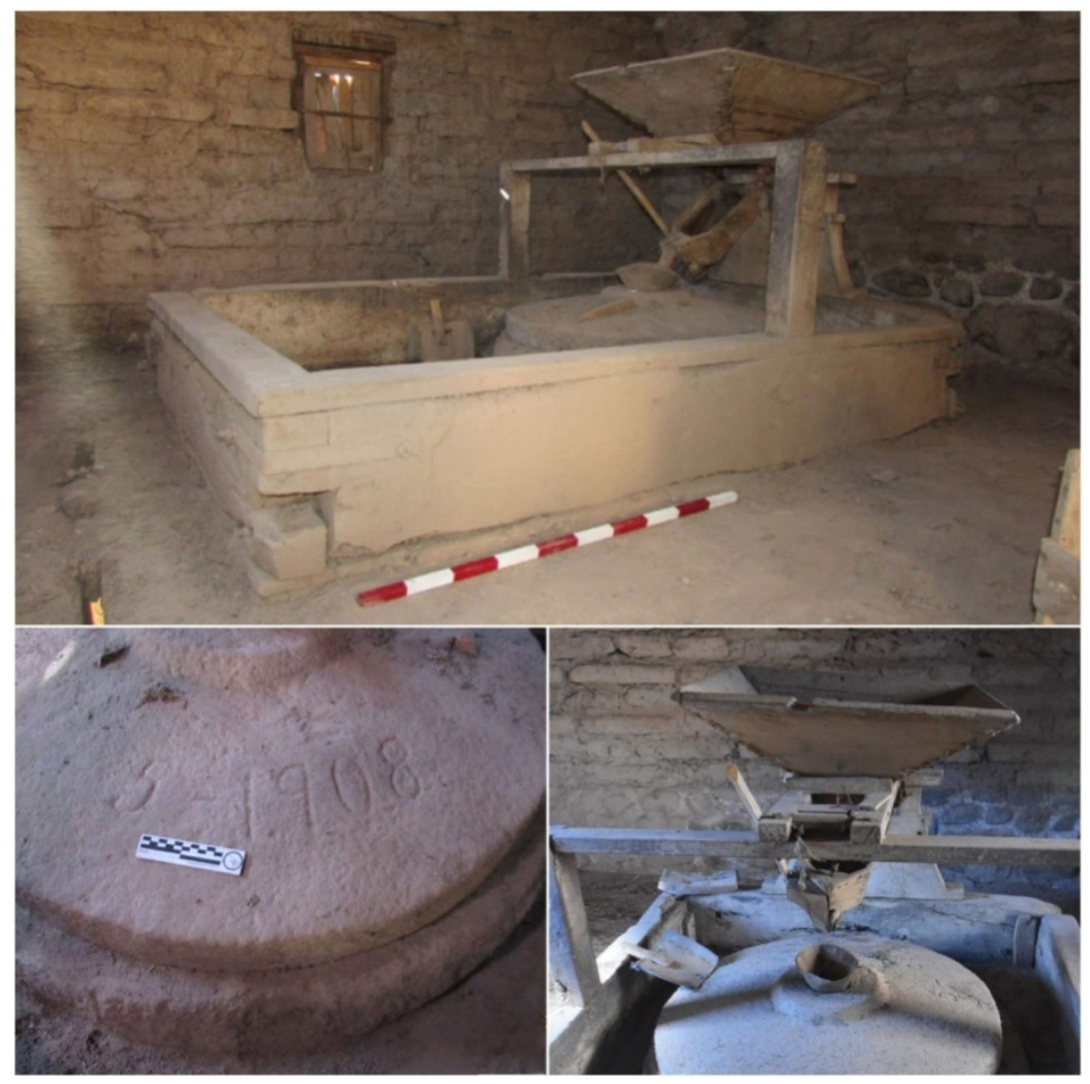

Figura 3. Diferentes partes de la maquinaria de molienda. Grabado de la fecha en la muela volantera.

En el proceso de molturación, la tolva debía estar abastecida de granos que luego pasaban a ser molidos por las muelas. Para que la muela superior se moviera era necesario el ingreso de agua por un canal con gran presión, en este caso una acequia que tomaba agua del río Calchaquí. La piedra de moler superior se movía a través de un rodezno o rodete (rueda con paletas en contacto directo con la fuerza hidráulica), el cual estaba conectado a la muela volantera a partir de un eje denominado árbol. Las muelas de moler trabajaban horizontalmente, al igual que el rodezno. El funcionamiento de estas estructuras ha sido descripto en detalle para los molinos de la región pampeana (Caggiano y Dubarbier, 2013;Sanmartin, 2013) y es posible homologarlo al de los molinos que se encuentran en el Noroeste argentino.

\section{Metodología}

Para abordar la cronología de funcionamiento del molino nos basamos en tres tipos de evidencias obtenidas a partir de diferentes vías metodológicas. Por un lado llevamos adelante una prospección inicial en las inmediaciones del molino harinero, con recolección superficial de materiales. Se hizo un relevamiento arquitectónico de las habitaciones del complejo (Figura 2) considerando dimensiones y características constructivas de las paredes, aberturas, cimiento y sobrecimiento. La información se consignó en planillas de datos cualitativos y cuantitativos, así como de registro fotográfico. Esta primera etapa de trabajo permitió seleccionar sectores a ser intervenidos estratigráficamente, a partir de los cuales 
se realizó una cuadrícula en la habitación E2 de 1 x $1 \mathrm{~m}$ de longitud, excavada por niveles artificiales de 0,05 m (ver Figura 2). Se obtuvieron 14 estratos artificiales hasta llegar al estéril; los materiales obtenidos incluyen: semillas, marlos de maíz, cerámicas, lozas, restos óseos, carbón, madera, papel, monedas, cuero y metal, que se encuentran actualmente en etapa de análisis.

Los materiales fueron tratados en el laboratorio, donde se procedió a la limpieza y conservación de los mismos. Las monedas fueron intervenidas con sucesivos baños en agua desmineralizada. Luego se colocaron individualmente en recipientes de plástico ideales para evitar la humedad, evitando así los cambios bruscos de temperatura y luminosidad. El papel fue limpiado cuidadosamente y guardado sin ningún tipo de intervención posterior que pudiera afectar su integridad.

Por otro lado durante los años 2017 y 2018 se realizó una revisión de diferentes fuentes documentales, específicamente en el "Archivo y Biblioteca Históricos Dr. Joaquín Castellanos” (en la sección de documentos históricos y Sala de Autores salteños) y en la "Biblioteca Provincial Atilio Cornejo" de la provincia de Salta. La fuentes obtenidas en dichos lugares fueron de capital importancia ya que brindan una exclusiva recopilación bibliográfica y documental especializada para provincia ya mencionada, con más de dos mil volúmenes librarios y unidades inexistentes en otros espacios, donde se pueden localizar apuntes históricos, descripciones de la industria, la economía, información estadística de la provincia, recopilación de leyes y memorias descriptivas llevadas a cabo por diferentes profesionales vinculados a organismos provinciales, producidos durante mediados del siglo XIX y principios del XX. Además se investigaron los Censos Nacionales que se encuentran digitalizados en la página de INDEC, que incluyen el Primer Censo Nacional de la República de 1869, el Primer Censo de Agricultura y Ganadería de 1888, el Segundo Censo Nacional de 1895, el Censo Nacional Agropecuario de 1908 y el Tercer Censo Nacional de 1914. Estas fuentes fueron analizadas teniendo en cuenta el contexto de la época y la subjetividad de los discursos propios de los momentos históricos en que fueron producidos (Nacuzzi y Lucaioli, 2011; Ricoeur, 1983).

Para finalizar se realizaron entrevistas informales no directivas (Guber, 2001) a los pobladores de Payogasta, seleccionando la información que aportaba a los objetivos de este trabajo, específicamente acerca del período de funcionamiento del molino y el momento que entró en desuso. Se consideraron miembros de la comunidad con conocimiento sobre el tema, que hubieran participado directamente en la dinámica de la molienda o que tuvieran conocidos o familiares que lo hubieran hecho.

\section{La utilización del molino: un siglo de molienda}

La información documental en relación a la actividad molinera para el departamento de Cachi es escasa, si bien hay datos que indican una temprana ocurrencia en Salta. De acuerdo a lo planteado por Nelson (1938), el primer molino hidráulico de la provincia data de 1582. Para el año 1881, la provincia ya contaba con 47 molinos instalados a lo largo de su territorio (Sola,1889), con una regulación que legislaba su funcionamiento e incluso preveía del valor de las harinas(Ojeda, 1866).

De acuerdo a lo planteado por Nelson (1938) el primer registro de un molino para el departamento de Cachi es de 1855. El documento obtenido en la "Biblioteca Provincial Atilio Cornejo" hace mención a un molino ubicado en Payogasta, propiedad de Hipólito Caro, que fue traspasado en 1865 al coronel Bonifacio Ruiz de los Llanos, quien había formado parte del Ejercito Auxiliar del Norte. En los terrenos que actualmente corresponden a la familia Ruiz de los Llanos también hay un molino hidráulico en desuso, por lo que no se puede concluir que las menciones documentales correspondan al molino que nos convoca. No obstante, esta información es un indicio de que hacia mediados del siglo XIX ya estaban

Aproximación cronológica al funcionamiento del molino hidráulico de Payogasta

P. J. Pifano y M. C. PÁEZ

(Cachi, Salta) durante los siglos XIX Y XX

Páginas 45-57 
presentes esta maquinarias en la zona. Para finales de este siglo, entre los municipios de Cachi, Lerma y Rosario sumaban siete molinos, cinco de los cuales eran movidos por rodezno (Sola, 1889). En el caso de Cachi, la producción de harinas habría alcanzado las 515 toneladas de trigo para el año 1894, siendo este producto uno de los más importantes, orientado al abastecimiento de las necesidades locales y regionales junto con los departamentos de Molinos y La Poma (Schlech, 1914; Pifano y Ermili,2020).

El relevamiento y registro arqueológico aportan datos cronológicos algo más precisos que la información documental en lo que respecta a finales del siglo XIX y gran parte del siglo XX. Al respecto, un primer dato a considerar se vincula con la fecha que figura grabada en la piedra de moler denominada volantera o móvil (Figura 3). La inscripción indica "5-1908” en su parte superior. Se trata de una muela que habría estado activa en el momento en que entró en desuso la estructura, dado que se encuentra perfectamente por encima de la muela fija o solera. No obstante, puede interpretarse que no sería la primera vinculada al molino ya que por fuera de la habitación de molienda, aunque en íntima relación espacial con ella, se encuentra otra piedra, con un desgaste notablemente superior. La excavación de uno de los recintos contiguos a la habitación de molienda ha aportado evidencia diagnóstica en términos cronológicos. Como ya se señaló, se han recuperado ítems cerámicos, óseos, líticos, fragmentos de loza, vidrio, madera, restos de cuero y papel, que actualmente se encuentran bajo análisis. No obstante, para este trabajo incorporaremos algunos resultados, diagnósticos a los fines de abordar la temporalidad del molino. Es el caso de tres monedas recuperadas en estratigrafía, una moneda obtenida de la recolección superficial en las inmediaciones del recinto y un fragmento de papel correspondiente a un documento electoral.

Dos de las monedas se encontraron en la unidad estratigráfica 1 (UE1), junto con el fragmento de papel, ambas asociadas a una estructura de madera muy deteriorada ubicada en una de las esquinas de la cuadrícula $(\mathrm{SW})$, además de un importante número de fragmentos de papel -dentro de los que se encuentran aquellos que corresponden a la boleta electoral-, restos arqueofaunísticos y un marlo de maíz. En la UE3 se encontró la tercera moneda, asociada igualmente a restos óseos y también a material antracológico.

Las cuatro monedas analizadas presentan variables adiciones de cloruros, típicos en materiales arqueológicos con cobre (focos pulverulentos verde claro), que se forman debido a que el cobre es un metal pesado de baja durabilidad, muy vulnerable al medio. Por ello el control de la humedad, dentro de la conservación preventiva, es fundamental ya que lo ataca muy rápido (López y Catalán,2001).

En la moneda 1 (UE1) se puede identificar el valor de 20 centavos en el centro, rodeado por laureles en el reverso. En el anverso se observa la "Cabeza de la Libertad con gorro frigio" diseñada por el grabador francés Eugene Oudine, rodeado por la inscripción República Argentina, dos estrellas y el año 1942 (Figura 4).

La moneda 2 (UE1) presenta un deterioro más marcado con incrustaciones de cloruros. A pesar de lo anterior se puede identificar su valor de 20 centavos en el centro (reverso), y el año 1924 en el anverso asociado a "La cabeza de la Libertad", al igual que la anterior (Figura 4). Tres fragmentos de papel que corresponden a un mismo documento electoral fueron encontrados en este nivel, donde se pudo identificar impreso el año 1973 y el mes de marzo, además del logo del Partido Justicialista y los candidatos a presidente y vicepresidente de entonces, Cámpora y Solano Lima, respectivamente (Figura 5).

La moneda 3 (UE 3) también presenta un deterioro importante. Si bien se identifica el valor de la misma en el reverso (20 centavos ubicados en el centro), el anverso está prácticamente borrado. Sin embargo a partir de su observación con lupa binocular pudimos inferir una posible fecha hacia finales de la década de 1890 (Figura 4). 


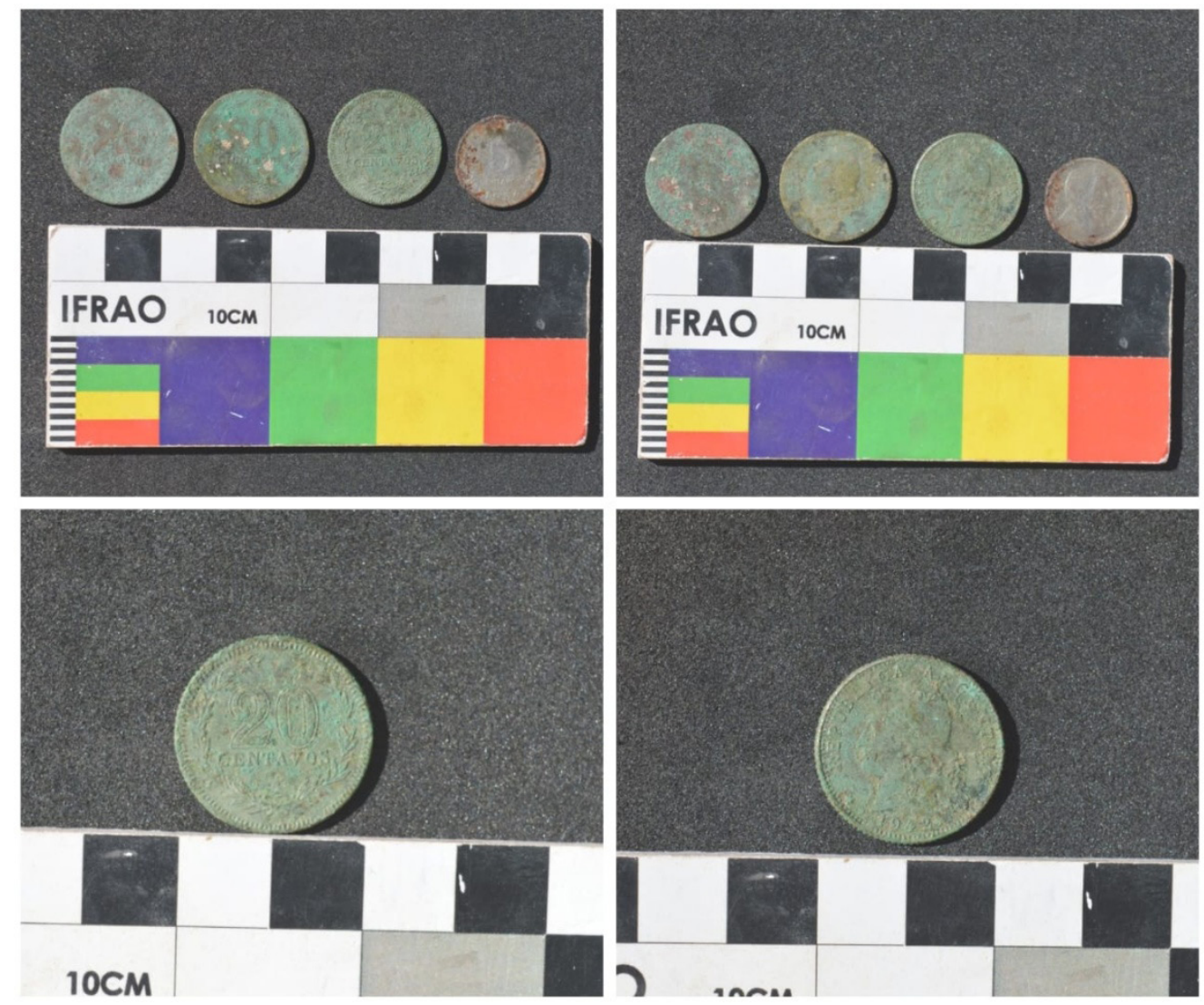

Figura 4. Sup. Izq. Reverso de las monedas ubicadas en orden cronológico, de izquierda a derecha: moneda 3 (década de 1890), moneda 2 (1924), moneda 1 (1942) y Moneda 4 (1954).

Sup. Der. Anverso de las monedas en el mismo orden. Inf. Izq.

Reverso de la moneda 1. Inf. Der. Anverso de la moneda 1.

Es importante mencionar que hacia diciembre de 1895 hubo una legislación nacional que establecía la acuñación de ejemplares de cuproníquel con una aleación de $75 \%$ de cobre y $25 \%$ de níquel. Las monedas de 20 centavos que aquí analizamos pertenecen a esta emisión y circularon desde 1896 a 1942 , con un peso de $4 \mathrm{gr}$, un diámetro de $21 \mathrm{~mm}$ y canto estriado (Fenoglio, 2010).

En cuanto a la moneda 4 (recolección en superficie), se observa la pátina de ambas caras a pesar de la presencia de óxido. En el reverso se encuentra el valor centro de 5 centavos rodeado por la inscripción "República Argentina" y el año 1954. En el anverso se ve el busto anciano San Martiniano con la inscripción “José de San Martin”(Figura 4). En 1950 apareció esta acuñación, conmemorando el aniversario de la muerte del prócer, que circuló hasta 1956. La moneda tiene un peso de 2 gramos, un diámetro de 17 mm y el canto estriado, siendo de acero bañado en cobre y níquel (Fenoglio, 2010).

Un aspecto a remarcar es la ubicación estratigráfica de las monedas. Es probable que la presencia en los tres primeros estratos de los 14 obtenidos, pueda explicarse a partir de la existencia de agentes tafonómicos, como es el caso de las cuevas de roedores, que se hicieron muy notorias en la UE4, y la abundancia de raíces desde la superficie hasta la UE7. Los hallazgos correspondientes a las monedas se encuentran en proximidades de ambas alteraciones, por lo que sería posible que su localización estrati- 
gráfica respondiera a una migración dentro del sedimento. No obstante, las características del contexto harían viable otra posible explicación. En la UE1 también se encontraron restos de cuero y madera. Los primeros conservan la forma de un contenedor, a manera de monedero, contiguo a la estructura de madera y a los fragmentos de papel de la UE1. Esto llevó a plantear, a manera de hipótesis, que las monedas podrían haber estado en el interior del contenedor de cuero, y que ambos (cuero y monedas) se podrían asociar a la madera de la misma UE. Siguiendo el mismo plano hipotético, es posible que la madera correspondiera a algún recipiente o cofre ubicado en las estanterías que aún presenta la habitación, y que su incorporación al contexto sedimentario aconteciera tardíamente, una vez que la estructura entró en desuso.

\section{EI desuso del molino en la memoria de los pobladores de Payogasta}

Durante las sucesivas intervenciones en el terreno, complementamos el trabajo arqueológico y de revisión documental con entrevistas informales a miembros de la comunidad de Payogasta, Bella Vista, Cachi y Molinos. A partir de las mismas abordamos diferentes temáticas que nos llevaron no sólo a identificar la importancia del molino para la memoria colectiva y la identidad de la comunidad local, sino también datos específicos sobre su funcionamiento, dinámica y transformaciones a lo largo del tiempo. En este sentido, las memorias se refieren generalmente a hechos históricos que han marcado el pasado de los pueblos, pero también la manera de cómo los recuerdan y los explican hoy en día, es decir cómo son experimentados estos sucesos históricos por determinadas colectividades (Cerda García, 2014).

En lo que respecta a los objetivos de este trabajo, es importante destacar que ninguno de los entrevistados recuerda con seguridad el origen del molino. Por ejemplo, uno de ellos, que en la actualidad reside en Salta capital pero vivió gran parte de su vida en Payogasta, dijo "(...) Yo he nacido en el 1948 y ya existía el molino así que debe ser de un poquito más antes, no sé qué año exacto puede ser (...)”. Otro de los pobladores sostiene que "(...)Sí, con respecto a cuándo dejo de funcionar si me acuerdo (...) Pero el año exacto que comenzó a funcionar ese molino no lo sé, pero debe ser mucho más antes que nazcamos nosotros $(\ldots)$ ".

Sin embargo, en cuanto al momento que dejo de utilizarse, todos lo sitúan hacia finales del siglo $\mathrm{XX}$ en relación con el ingreso y consumo generalizado de la harina blanca o comercial. Las fechas concretas aportadas por los entrevistados corresponden a 1970, 1979, 1980, 1982 y 1985. Una cuestión a considerar es que el último molinero que habría trabajado allí falleció en 1998, lo que guarda relación con las fechas precedentes. Por su parte, la evidencia arqueobotánica recuperada de las piedras de moler de la estructura indican que se habría molido pimiento, un tipo de cultivo de renta que se incorporó al valle en 1937 (Manzanal, 1987 y 1995; Mariangeli et al. 2016; Pais, 2011), lo que apoya la idea de que el desuso podría situarse entre las décadas de 1970 y 1980, habiendo funcionado, tal vez ocasionalmente, para la molienda este producto.

Aproximación cronológica al funcionamiento del molino hidráulico de Payogasta

P. J. Pifano y M. C. PÁEZ

(Cachi, Salta) durante los siglos XIX y XX

Páginas 45-57 


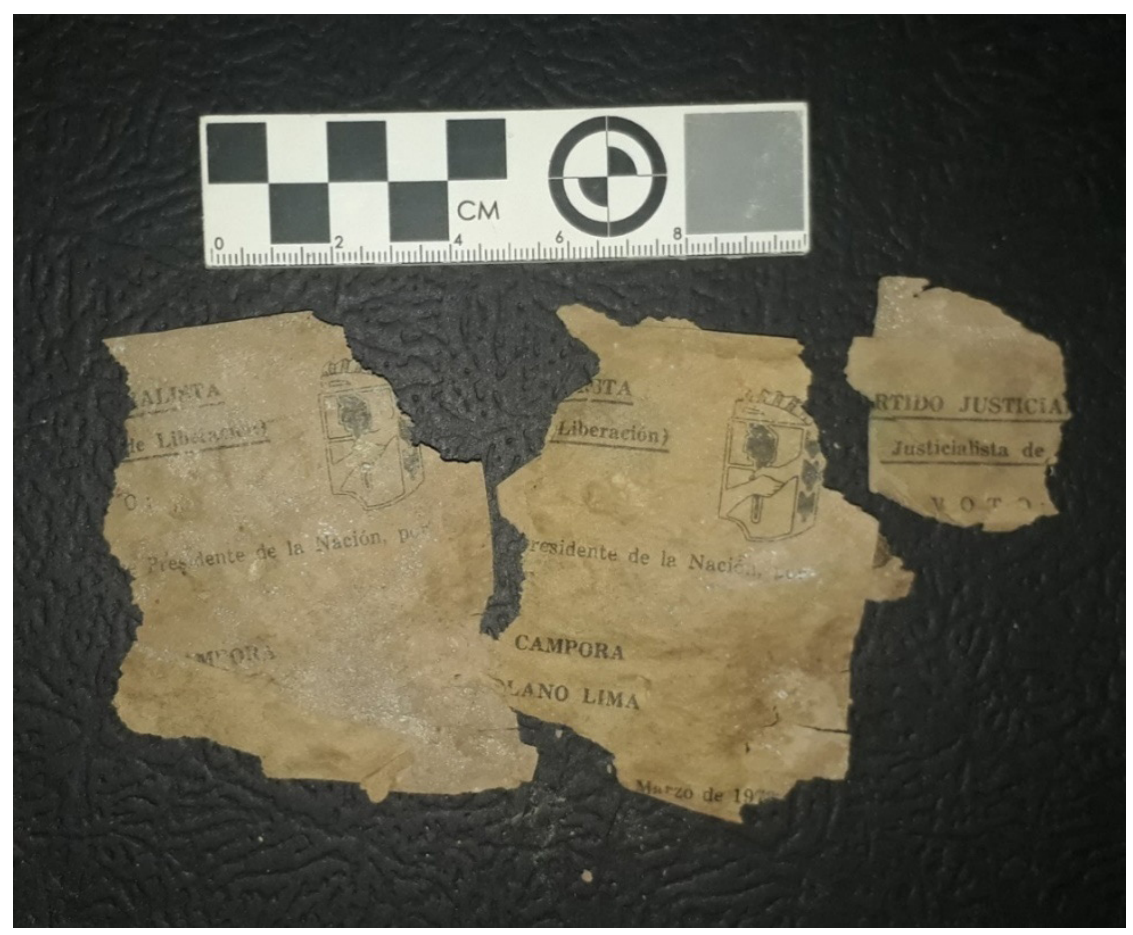

Figura 5. Fragmentos de papel correspondientes a una boleta electoral de 1973.

\section{Discusión y conclusiones}

La problemática temporal del molino de Payogasta representa un interés primario en el marco de una investigación mayor referida a la dinámica de molienda en el Valle Calchaquí. El primera instancia trabajamos desde la arqueología, no obstante tempranamente pudimos identificar limitaciones para alcanzar resultados precisos sin la colaboración de otras disciplinas. Este abordaje, sin embargo, no implicó una sumatoria de métodos sino, por el contrario, indagar integralmente con los alcances, limitaciones y tensiones que existen en y entre cada uno de ellos desde una perspectiva holística. En este sentido, uno de los primeros elementos a destacar en torno a esta investigación tiene que ver con las ventajas de utilizar un enfoque transdisciplinario para abordar (y comprender) la temporalidad en tanto problema de investigación.

En base a ello, debemos discutir aspectos que conciernen al momento en que comenzó a funcionar el molino. La información procedente de la excavación arqueológica, si bien, como mencionamos, no se ha terminado de analizar, no nos permite llegar a una fecha precisa. El dato inequívoco más directo es aportado por la muela volantera que forma parte de la maquinaria, que tiene grabada la fecha 5-1908. No obstante, hay otros elementos que nos permiten inferir que la utilización podría remontarse a algunas décadas previas. Por un lado, la información documental que menciona que para 1855 ya había estructuras similares en Payogasta. Por otro, la presencia de una muela volantera a la entrada del molino, cuyo notable desgaste sugeriría que funcionó con anterioridad a 1908. El hallazgo de una moneda en estratigrafía en la habitación excavada, con una inscripción de la década de 1890 respalda esta propuesta.

En virtud de ello, es posible inferir que la actividad en el molino se habría iniciado hacia la segunda 
mitad del siglo XIX, cronología que explica de alguna manera la falta de registro en la memoria colectiva de los pobladores de Payogasta. Es probable que lo que se transmitió a partir de la oralidad de dos generaciones atrás -inicios del siglo XX-, sea el recuerdo de un molino ya en pleno funcionamiento.

La utilización durante el siglo XX parece mostrar continuidad temporal. Las monedas nos sitúan en 1924, 1942 y 1954, y la boleta electoral en 1973. Esta última fecha concuerda con la información procedente de los registros orales y la evidencia arqueobotánica de pimiento en la piedra de moler. Es posible que en los últimos años de funcionamiento del molino hubiera una molienda ocasional de este producto, poco significativa para la memoria colectiva de los pobladores locales, a juzgar por las escasas menciones en los relatos orales. Según datos de la Subsecretaría de Industria y Mercados, en la actualidad hay dos molinos industriales en el departamento de Cachi que empezaron a funcionar en la década de 1990. Es probable que la falta de acuerdo entre los entrevistados acerca del desuso de la estructura en cuestión, si bien las fechas se mantienen en un rango de dos décadas -'70 y '80-, pueda explicarse a partir de un uso muy esporádico durante estos años que se interrumpió definitivamente con el auge de los molinos industriales. Este y otros aspectos que adquieren validez en tanto hipótesis de trabajo podrán ser profundizados en futuras investigaciones.

\section{Agradecimientos}

En primer lugar a Ariel López Miranda y su familia, a la gente de la comunidad de Payogasta, a las autoridades del Museo Antropológico de Salta y del Museo Pío Pablo Díaz de Chachi, a CONICET que proporciono el financiamiento para los trabajos de campo, al equipo de trabajo del laboratorio 103 que participó de los trabajos de campo y a Agustín Betz y Julieta Igoa que colaboraron en la elaboración de los mapas y figuras. Finalmente, a los revisores anónimos que permitieron mejorar la versión original del manuscrito. La responsabilidad por lo expresado, no obstante, es exclusiva de los autores.

\section{Notas}

${ }^{1}$ Tesis en realización en la Facultad de Ciencias Naturales y Museo, Universidad Nacional de La Plata, titulada "TERRITORIO Y PROCESO SOCIAL EN LA PRODUCCIÓN HARINERA EN EL SIGLO XIX EN EL VALLE CALCHAQUÍ (SALTA, ARGENTINA)” cuyo objetivo es analizar la dinámica de producción harinera durante el siglo XIX en el sector norte del Valle Calchaquí (Cachi, Salta).

\section{Referencias bibliográficas}

CAGGIANO, M. A., Y DUBARBIER, V. (2013). Elementos modeladores del paisaje natural y cultural en La Pampa Chivilcoyana: La introducción del cultivo de trigo. Anuario de Arqueología, Vol 5, 213-230.

CASTRO-GÓMEZ, S. (2007). Decolonizar la universidad. La hybris del punto cero y el diálogo de saberes”. En R.Grosfoguel y S. Castro-Gómez (Eds.), El giro decolonial. Reflexiones para una diversidad epistémica más allá del capitalismo global, pp. 79-91. Bogotá: Siglo del Hombre Editores.

CERDA GARCÍA, A. (2014). Memorias largas y cortas: tensiones para su articulación en el campo indígena. Clepsidra. Revista Interdisciplinaria de Estudios sobre Memoria1, 82 - 89.

CIEZA, G. (2010). Procesos organizativos y acceso a la tierra en el Valle Calchaquí (Tesis de Maestría).

Aproximación cronológica al funcionamiento del molino hidráulico de Payogasta

P. J. PifAnO Y M. C. PÁEZ

(Cachi, Salta) durante los siglos XIX y XX

Páginas 45-57 
Facultad de Ciencias Agrarias y Forestales, Universidad Nacional de La Plata.Argentina.

FENOGLIO, J.(2010). Billetes y Monedas de Argentina. Recuperado de:https://legislaturalarioja.gob.ar/ documentos/Argentina-Fenoglio-2010.pdf.

GUBER, R (2001).La etnografía, método, campo y reflexividad. Bogotá: Grupo Editorial Norma.

HOCSMAN, L. (2003). Estructura rural, territorialidad y estrategias domésticas en la Cordillera Oriental (Tesis de Doctorado). Facultad de Ciencias Naturales y Museo, Universidad Nacional de La Plata. Argentina.

INDEC (Instituto Nacional de Estadística y Censos) (1869). Primer Censo Nacional de la República. Buenos Aires. Argentina.

INDEC (Instituto Nacional de Estadística y Censos) (1888). Primer Censo de Agricultura y Ganadería. Buenos Aires. Argentina.

INDEC (Instituto Nacional de Estadística y Censos) (1895). Segundo Censo Nacional de la República. Buenos Aires. Argentina.

INDEC (Instituto Nacional de Estadística y Censos) (1908). Segundo Censo Nacional. Agropecuario. Buenos Aires. Argentina.

INDEC (Instituto Nacional de Estadística y Censos) (1914). Tercer Censo Nacional de la República. Buenos Aires. Argentina.

LERA, M. E. (2005). Transformaciones económicas y sociales en el departamento de Cachi (Salta) a fines del Siglo XIX (Tesis de Licenciatura). Facultad de Humanidades, Universidad Nacional de Salta. Argentina.

LÓPEZ G. Y CATALÁN E. (2001).Limpieza y criterios de conservación para monedas y medallas.Museo Casa de la Moneda, pp.79-85.Madrid, España.543. Recuperado de comon.mini.icom.museum/ wp-content/uploads/sites/20/2018/12/Cleaning_and_preservation_criteria_for_coins_and_medals. pdf.

MANZANAL, M. (1987). Pobreza y marginalidad en el agro argentino: la producción agrícola y su comercialización en Cachi, Salta, Cuadernos del CEUR, $N^{\circ}$ 20, 1-120.

MANZANAL, M. (1995). Globalización y ajuste en la realidad regional argentina: ¿reestructuración o difusión de la pobreza?Realidad Económica 134: 67-82.

MARINANGELI， G. A., PLASTINÉ PUJADAS I. G. Y PAEZ, M.C. (2016). Aproximaciones a las transformaciones productivas en el norte del Valle Calchaquí (depto de Cachi Salta). En Actas de las VIII Jornadas de investigación en Antropología Social Santiago Wallace, pp. 1987-1997. Buenos Aires.

MARINANGELI, A. G. Y PÁEZ, M. C. (2019). Transformaciones en la organización agrícola de pequeños productores del Valle Calchaquí norte (departamento de Cachi,Salta). Diálogo Andino58 (1), 101-113. 
Año IX, Volumen 10 | 2020 / ISSN en línea: 2591-2801 | ISSN versión impresa: 2250-866X

MARTÍNEZ MIGUÉLEZ, M. (2007). Conceptualización de la transdisciplinariedad, Polis 16. Recuperado en: http://journals.openedition.org/polis/4623

MATA DE LÓPEZ, S. E. (2005). Tierra y poder en Salta. El noroeste argentino en vísperas de la independencia. Salta: CEPIHA.

MOLINA Y VEDIA, S. (2016). Metodología del proyecto transdisciplinario "Las formas del cambio", Memoria Académica. Recuperado en: http://www.memoria.fahce.unlp.edu.ar/trab_eventos/ ev.8514/ev.8514.pdf

NACUZZI, L., Y LUCAIOLI,C. (2011). El trabajo de campo en el archivo: campo de reflexión para las Ciencias Sociales. Publicar $I X \mathrm{~N}^{\circ} \mathrm{X}, 47-62$.

NELSON, C. (1938). Boletín del Instituto San Felipe y Santiago de estudios históricos de Salta. Número uno. Notas sobre la industria harinera en salta. Biblioteca Provincial Atilio Cornejo. Provincia de Salta. Argentina.

OJEDA, G. (1866). Recopilación general de leyes de la Provincia de Salta y sus decretos regulatorios. Tomo 1. Archivo y Biblioteca Históricos Dr. Joaquín Castellanos (sección documentos históricos y Sala de Autores salteños). Provincia de Salta. Argentina.

PAIS, A. L. (2011). Las transformaciones en las estrategias de reproducción campesinas en tiempos de globalización. El caso de Cachi en los Valles Calchaquies(Tesis de Doctorado). Facultad de Ciencias Agropecuarias, Universidad Nacional de Córdoba. Argentina.

PIFANO, P. J Y DABADIE, M. (2016). Approach to the Grist Milling Activity in Northern Calchaqui Valley (Salta) during the 19th and 20th Centuries. The International Journal Of Humanities \& Social Studies 4 (6), 326-333.

PIFANO, P. J Y ERMILI, L. (2020) La producción harinera en el norte de los Valles Calchaquies (Provincia de Salta, Argentina). Las fuentes documentales y el molino harinero de Payogasta. MS.

RICOEUR, P (1983). Freud: una interpretación de la cultura. México: Siglo XXI.

SCHLECH, E (1914). Salta y sus riquezas apuntes económicos y estadísticos. Buenos Aires. Archivo y Biblioteca Históricos Dr. Joaquín Castellanos (sección documentos históricos y Sala de Autores salteños). Provincia de Salta. Argentina.

SANMARTÍN, L. (2013). Patrimonio industrial, molinos y hangares. En IAA/ Seminario de Crítica Número 183. Recuperado en: http://www.iaa.fadu.uba.ar/publicaciones/critica/0183.pdf

SOLA, M (1889). Memoria descriptiva de la Provincia de Salta. Archivo y Biblioteca Históricos Dr. Joaquín Castellanos (sección documentos históricos y Sala de Autores salteños). Provincia de Salta. Argentina.

Recibido: 2 de Mayo 2020

Aceptado: 16 de Julio 2020

Aproximación cronológica al funcionamiento del molino hidráulico de Payogasta

P. J. Pifano y M. C. PÁEZ

(Cachi, Salta) durante los siglos XIX y XX

Páginas 45-57 